\title{
Fortschritte in der Gesichtstransplantation
}

\author{
Progress in Face Transplantation
}

Autoren

Institute

\section{Kueckelhaus ${ }^{1,2}$, M. Lehnhardt' ${ }^{2}$, S. Fischer ${ }^{1}$, E. Eriksson' ${ }^{1}$, B. Pomahac ${ }^{1}$, T. Hirsch ${ }^{2}$}

${ }^{1}$ Division of Plastic Surgery, Brigham and Women's Hospital, Harvard Medical School, Boston, United States ${ }^{2}$ Klinik für Plastische Chirurgie und Schwerbrandverletzte, Handchirurgiezentrum, Operatives Referenzzentrum für Gliedmaßentumore, BG Universitätsklinik Bergmannsheil, Bochum

\author{
Schlüsselwörter \\ - experimentelle Transplan- \\ tation \\ - Koaptation \\ - kraniofaziale Chirurgie \\ - Gesicht \\ - Mikrochirurgie
}

\section{Key words}

- experimental transplantation

- coaption

- craniofacial surgery

- face

- microsurgery

eingereicht 20.3.2014 akzeptiert 9.7.2014

Bibliografie DOI http://dx.doi.org/ 10.1055/s-0034-1385850 Handchir Mikrochir Plast Chir 2014; 46: 206-213

(c) Georg Thieme Verlag KG Stuttgart · New York ISSN 0722-1819

Korrespondenzadresse Prof. Bohdan Pomahac Division of Plastic Surgery Brigham and Women's Hospital Harvard Medical School Boston

United States

bpomahac@partners.org

\section{Zusammenfassung}

Die Vascularised Composite Allostranspantation (VCA) dient zur Restoration komplexer Defekte. Restoration beschreibt in diesem Zusammenhang die Wiederherstellung von destruiertem Gewebe durch anatomisch identische Strukturen. Bis heute sind weltweit insgesamt bereits über 150 VCAs, davon 31 Gesichtstransplantationen, durchgeführt worden. Die Gesichtstransplantation stellt keine primär lebenserhaltende Maßnahme dar, sondern dient der Verbesserung der Lebensqualität betroffener Patienten. Aufgrund der sich zwingend anschließenden lebenslangen Immunsuppression und den damit verbunden potentiell lebensbedrohlichen Nebenwirkungen ist eine äußerst strenge Indikationsstellung mit individueller Bewertung des Kosten-Nutzen-Verhältnisses essentiell. Sichere Revaskularisation und möglichst vollständige ästhetische und funktionelle Reintegration sind die ultimativen Ziele der Gesichtstransplantation. Aktuell werden, je nach Zentrum, unterschiedliche immunsuppressive Strategien zur Induktions- und Erhaltungstherapie nach Transplantation angewandt. Die Haut bedarf als immunogenste Komponente des Transplantats weiterer Forschung, um mit den Erfolgen der immunsuppressiven Therapie bei transplantierten soliden Organen gleichziehen zu können. Auch die Organpreservation während des Transfers von Spender zu Empfänger stellt ein wichtiges Forschungsgebiet dar. Mit Publikation motivierender funktioneller und ästhetischer Ergebnisse hat sich die zunächst negative Einstellung hinsichtlich dieser nicht überlebensnotwendigen Transplantationen zum Positiven gewandelt. Kontinuierliche Weiterentwicklung chirurgischer Techniken und immunsuppressiver Strategien bilden die Grundlage zur erfolgreichen Etablierung dieses spannenden jungen Verfahrens.

\section{Abstract}

Vascularised composite allotransplantation (VCA) is utilised for restoration of complex defects. In this context, restoration describes the replacement of destroyed tissue by identical anatomic structures. Up to date, over 150 VCAs including 31 face transplantations have been performed worldwide. Face transplantation is a life giving, rather than life saving procedure that is intended to significantly improve the patient's quality of life. Safe revascularisation as well as aesthetic and functional reintegration are the ultimate goals of face transplantation. The necessary lifelong immunosuppression with potentially life-threatening side effects imposes the need for a very strict risk-benefit ratio assessment and currently limits the indications of face transplantation. Different transplant centres use different protocols for induction and maintenance immunosuppression. Skin is the most immunogenic part of the vascularised composite allograft and has been the focus of intensive research efforts in order to replicate the success of immunosuppressive regimens for solid organ transplantation. Organ preservation during transfer from donor to recipient is another important field of research within VCA. The general public's originally rejecting attitude towards non-lifesaving VCA procedures has changed towards a general acceptance following the publication of promising results after the first cases of face transplantation. Further improvements of surgical techniques and immunosuppressive strategies will be important to drive these young and exciting procedures forward in the future. 


\section{Einleitung \\ $\nabla$}

Die Behandlung von Verletzungen, die mehrere Schichten funktionellen Gewebes betreffen, ist eine der großen Aufgaben der plastischen Chirurgie. Aufgrund der Tatsache, dass der konventionellen Rekonstruktionschirurgie bei ausgeprägten Defekten komplexer Strukturen sowohl in ästhetischer wie funktioneller Hinsicht Grenzen gesetzt sind, hat sich das Gebiet der Vascularized Composite Allotransplantation (VCA) entwickelt. Als Composite Tissue, also „zusammengesetztes Gewebe“, werden Strukturen bezeichnet, die sich aus verschiedenen Grundgeweben zusammensetzen. So beinhalten Gesicht und Extremitäten bspw. Muskeln, Haut, Knochen, Sehnen, Knorpel, Gefäße, Nerven und andere Gewebe. VCA definiert in diesem Zusammenhang die Transplantation einer vitalen, aus unterschiedlichen Geweben zusammengesetzten, funktionellen Einheit (z. B.: Hand oder Gesicht) von einem Spender zu einem Empfänger. Bis heute sind weltweit über 150 VCAs zur Rekonstruktion verschiedenster anatomischer Strukturen, wie Gesicht, obere und untere Extremitäten, Larynx, Trachea und Bauchdecke, durchgeführt worden.

Während des zweiten Weltkrieges wurden Brandverletzungen mit allogenen Hauttransplantaten versorgt. Deren Abstoßung führte zu den ersten Untersuchungen in der Transplantationsimmunologie [1-3]. Angetrieben von den ersten Studien über Hauttransplantatabstoßung, führte der plastische Chirurg Joseph Murray in Boston im Jahre 1954 die erste erfolgreiche klinische Organtransplantation von Nieren zwischen eineiigen Zwillingen durch [4]. Im Jahre 1959 folgte dann unter Anwendung von Ganzkörperbestrahlung die erste Nierentransplantation zwischen 2-eiigen Zwillingen [5]. Es sollte nur noch bis zum Anfang der 60er Jahre dauern, bevor Steroide und Azathioprin als erste chemische Immunsuppressiva die allogene Nierentransplantation ermöglichten [6]. Die erste dokumentierte VCA war der Versuch einer Handtransplantation im Jahre 1963 [7,8]. Diese scheiterte aufgrund einer akuten Abstoßungsreaktion nach 2 Wochen mit konsekutivem Transplantatverlust. Das stetig wachsende Verständnis über das menschliche Immunsystem und der immense Fortschritt in der Entwicklung von Strategien zur Immunsuppression über die folgenden Jahrzehnte führte schließlich, über 3 Jahrzehnte nach dem ersten Versuch, zur ersten erfolgreichen Handtransplantation im Jahre 1998 in Lyon, Frankreich $[9,10]$.

Ebenfalls in Frankreich in Amiens wurde im Jahre 2005 die erste partielle Gesichtstransplantation durchgeführt [11]. Die ästhetischen und funktionellen Ergebnisse dieses ersten Versuchs motivierten weitere Zentren zur Gründung eigener Transplantationsprogramme. Bis Februar 2014 ist weltweit insgesamt über 31 partielle oder totale Gesichtstransplantationen berichtet worden [12,13]. Über einige Fälle ist bis dato noch nicht in der Fachpresse, sondern lediglich in öffentlichen Medien, berichtet worden. 6 Gesichtstransplantationen (2 partielle und 4 totale) sind am Transplantationszentrum des Brigham and Women's Hospital in Boston, USA durchgeführt worden ( $\bullet$ Abb. 1).

\section{Rekonstruktion oder Restoration}

$\nabla$

Das Gesicht ist ein einzigartiges Organ. Obwohl es viele Funktionen wie Atmung, Sprachproduktion und Essensverarbeitung unterstützt, ist das reine Erscheinungsbild an sich seine eigentliche Hauptfunktion. Gesichtserkennung wird als angeborene mensch-

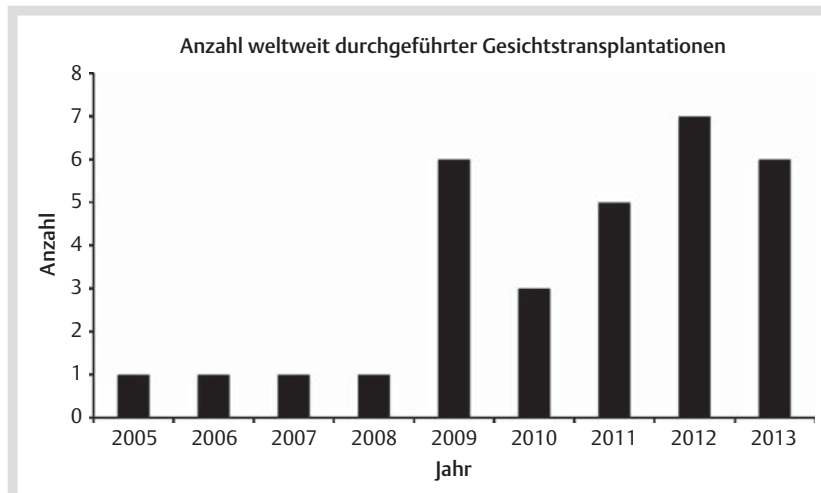

Abb. 1 Anzahl weltweit durchgeführter Gesichtstransplantationen, Stand Februar 2014

liche Fähigkeit angesehen. Die starren Gesichtsstrukturen dienen hierbei zur Identifikation einzelner Personen, die Bewegungsfähigkeit spielt eine zentrale Rolle in der sozialen Kommunikation [14]. Obwohl die Restoration nach partiellem oder totalem Verlust des Gesichts und seiner Funktionen in den meisten Fällen keine direkt überlebenssichernde Maßnahme darstellt, so steht der Gewinn an Lebensqualität für die Patienten im Vordergrund. Bei schweren Gesichtsverletzungen, Verbrennungen und ausgedehnten Tumorresektionen sind manchmal mit konventionellen rekonstruktiven Maßnahmen keine zufriedenstellenden Ergebnisse realisierbar [15]. In diesen Fällen gilt es die potentielle Verbesserung der Lebenssituation durch Restoration mittels VCA gegen die damit verbundenen Risiken abzuwägen. Eine VCA und damit Restoration beschreibt in diesem Zusammenhang den Ersatz des zerstörten Gewebes durch anatomisch identische Strukturen, Rekonstruktion das Mobilisieren anderer anatomischer Strukturen zum Ort der Verletzung hin. Zwar können durch rekonstruktive Maßnahmen Strukturen wie die Nase nachempfunden werden, jedoch sind zur vollen Reintegration des Gesichts weitaus komplexere Funktionen notwendig. Hierzu gehört die Motorik von Lippen und Augenlidern sowie Gesichtsmuskeln. Da wir unsere Gesichter im täglichen Leben nur selten selbst sehen, ist die Oberflächensensibilität sowie die Propriozeption für die Interaktion mit der Umwelt essentiell. Somit spielt sowohl die motorische als auch die sensible Innervation für den Erfolg der Reintegration des Transplantats eine wichtige Rolle. Es konnte gezeigt werden, dass die Koaptation von Hauptästen der sensiblen und motorischen Nerven zu einer kortikalen Wiedereingliederung im Motorkortex führen kann und somit auch eine funktionelle Rehabilitation des Gesichts ermöglicht [16]. Folglich ist also nicht nur die Motorik, sondern auch das sensible Feedback entscheidend für bessere funktionelle Ergebnisse.

\section{Patientenscreening}

Potentielle Empfänger für ein Gesichtstransplantat werden äußerst genau und nach strengen Kriterien evaluiert, um sicherzustellen, dass das zu erwartende Outcome die Risiken des Eingriffs überwiegt. Der potentielle Empfänger muss sich der Risiken und Nebenwirkungen der sich anschließenden lebenslangen Immunsuppression bewusst sein. Zudem muss für jeden Patienten individuell festgestellt werden, ob die Operation sicher durchgeführt werden kann und der Empfänger realistische 
Erwartungen an das Ergebnis hat. Der Screeningprozess ist eine multidisziplinäre Aufgabe, in die unter anderem plastische Chirurgen, Transplantationsimmunologen, Radiologen, Mikrobiologen, Psychologen, Sozialarbeiter und Physiotherapeuten in enger Zusammenarbeit involviert sind. Nachdem das Chirurgenteam das Ausmaß des Defekts und die Durchführbarkeit der Transplantation bewertet hat, erstellen die Transplantationsimmunologen ein Profil des potentiellen Empfängers. Hierbei spielen unter anderem Blutgruppe, HLA (humanes Leukozytenantigen)Profil und bestehende Sensibilisierung gegen bestimmte Antigene eine Rolle. Die Radiologen erstellen ein Gesamtbild der anatomischen Gegebenheiten. Mittels CT und Angiografie werden die tiefen Strukturen der Kopf- und Halsregion sowie die Gefäßanatomie dargestellt. Das MRT dient zur Bewertung der Weichteilsituation. Am Transplantationszentrum des Brigham and Women's Hospital in Boston wird zusätzlich präoperativ ein FunktionsMRT durchgeführt, um postoperative kortikale Umbauprozesse im Rahmen der funktionellen Reintegration des Transplantats besser beurteilen zu können. Der Patient wird auf Hepatitis A, B und C, Zytomegalievirus (CMV), Varizellen (VZV), Epstein Barr Virus (EBV), HIV, Tuberkulose, Toxoplasmose und Syphilis getestet und präoperativ mit notwendigen Impfungen versorgt. Die psychologische Beurteilung darf neben den üblichen Stressfaktoren der prä-, peri- und postoperativen Maßnahmen auch die Belastungen durch das zu erwartende Medieninteresse nicht außer
Acht lassen. Sozialarbeiter analysieren und stabilisieren das soziale Umfeld, sowohl in der präoperativen als auch postoperativen Phase. Die Physiotherapeuten arbeiten zusammen mit dem Patienten schon vor der Transplantation ein intensives Trainingsprogramm aus, das neben Umfang und Frequenz auch Ziele und Erwartungen des Kandidaten beschreibt. Der Patient muss zum Erreichen eines möglichst optimalen funktionellen Ergebnisses diesen Therapieplan voll und ganz unterstützen ( $\bullet$ Abb. 2).

\section{Problem Organtransfer \\ $\nabla$}

Ein wichtiger Punkt zum Erreichen einer breiteren Anwendung der Gesichtstransplantation und VCAs im Allgemeinen ist die Verlängerung der maximal möglichen Ischämiezeit und die Prozessoptimierung im Rahmen des Organtransfers. Muskelgewebe reagiert im Vergleich mit soliden Organen wie Niere und Leber extrem empfindlich auf Sauerstoffmangel und stellt den sensitivsten Teil des Gesichtstransplantats dar. Während Nieren eine kalte Ischämiezeit von 36-48 h [17] in Preservationslösung tolerieren und für die Leber immerhin noch $12-18 \mathrm{~h}$ [18] gelten, so werden für Herztransplantationen generell noch immer nur $4 \mathrm{~h}$ kalte Ischämie empfohlen [19]. Da Gesichtstransplantate in der Regel ebenfalls eine signifikante Masse an Muskelgewebe enthalten, geht man auch hier von einer nicht zu überschreitenden
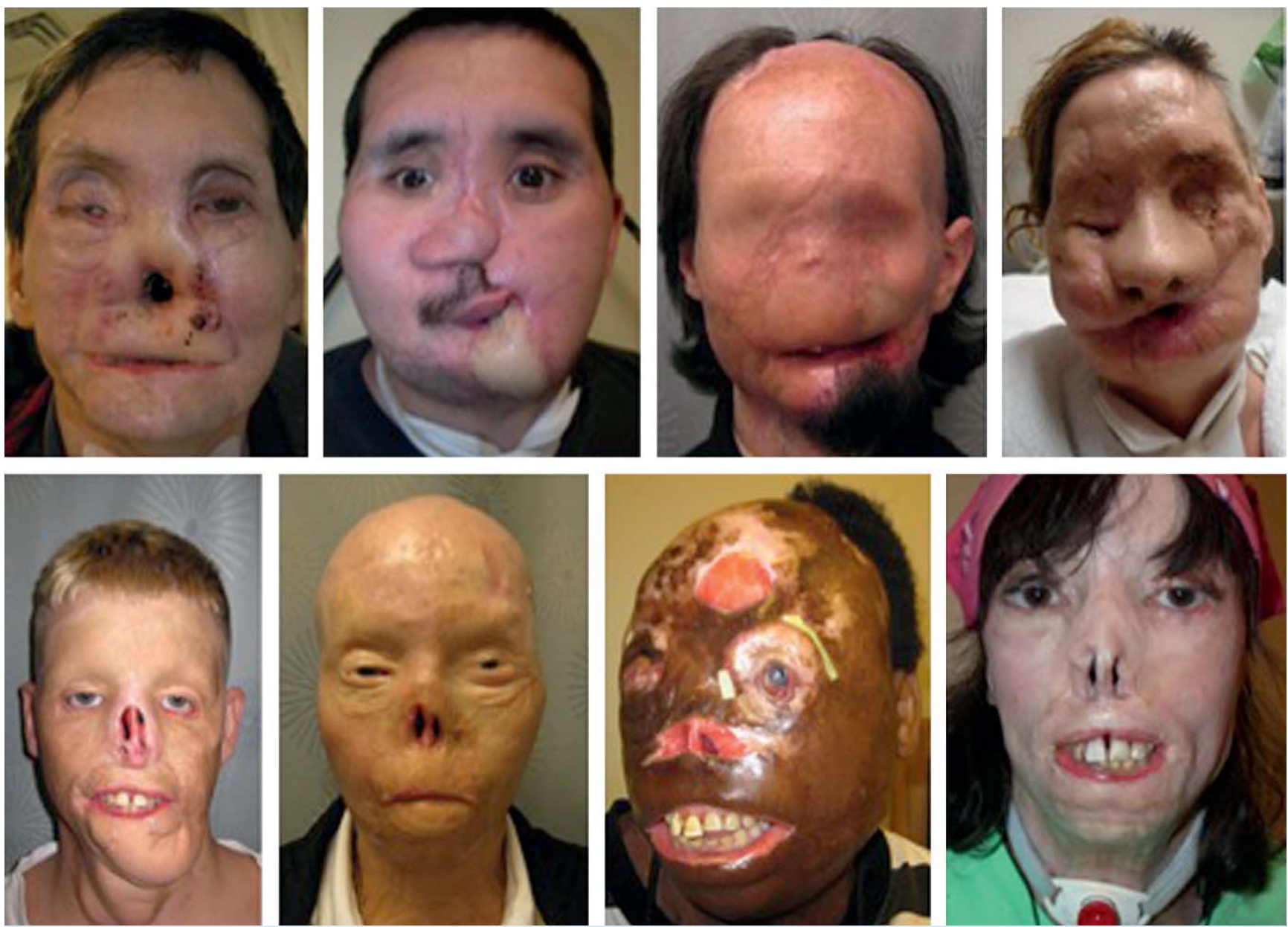

Abb. 2 Frontalansicht von 8 Transplantationskandidaten. Nach Ausschöpfung konventioneller rekonstruktiver Maßnahmen bestand bei allen Patienten aufgrund fortbestehender ausgeprägter funktioneller und ästhetischer Einschränkungen die Indikation zur Transplantation. Publiziert in: Evolution of indications for facial transplantation. Pomahac et al. J Plast Reconstr Aesthet Surg. 2011 Nov; 64 (11): 1410-6. doi: 10.1016/j.bjps.2011.06.024. Epub 2011 Aug 16.PMID: 21843979. 
kalte Ischämiezeit von 4 Stunden aus [20]. Die Kürze dieser Zeitspanne schränkt wiederum den Spenderradius und damit die Organangebote pro Patient auf der Warteliste stark ein. Um diesem Dilemma zu entkommen gibt es mindestens 2 Lösungsansätze: Auf der einen Seite gilt es die Abläufe der Transplantathebung und -implantation sowie die Orchestrierung der dazu notwendigen Chirurgenteams zu optimieren, um kostbare Ischämiezeit einzusparen. Auf der anderen Seite sollte die Erforschung alternativer Preservationsmethoden, wie die Transplantatperfusionmit transportablen extrakorporalen Membranoxygenierungs(ECMO)-maschinen weiter vorangetrieben werden, um mögliche Ischämiezeit hinzuzugewinnen. Ein Zeitgewinn kann dabei zu einer exponentiellen Erweiterung des Spenderradius führen.

\section{Operative Technik \\ $\nabla$}

Die ultimativen Ziele der Gesichtstransplantation sind die sichere Revaskularisation des transplantierten Gewebes sowie die möglichst vollständige Reintegration des Gesichts, sowohl in ästhetischer als auch funktioneller Hinsicht. Da jede einzelne Gesichtstransplantation ein individuelles Vorgehen erfordert, werden im Folgenden die wesentlichen einheitlichen Aspekte der operativen Technik dargestellt ( $\bullet$ Abb. 3).

Die A. facialis stellt die Versorgung der Weichteile des Gesichts sicher. Sie besitzt ein ausgedehntes Netzwerk von Anastomosen mit der A. carotis interna. Über die A. angularis im inneren Augenwinkel kommuniziert die A. facialis mit den Ästen der A. ophthalmica der A. carotis interna. Darüber hinaus befinden sich multiple Anastomosen zwischen der A. facialis und der A. maxillaris über A. orbitalis inferior und A. mentalis [15]. Die klinische Erfahrung aus ästhetischen und rekonstruktiven Gesichtsseingriffen zeigt, dass ausgedehntes Unterminieren im subkutanen Gewebe im Gesichtsbereich sicher durchgeführt werden kann. Relativ gefahrloses Präparieren in tieferen Schichten direkt superfiziell der Fascia parotidea, wie es für die Gesichtstransplantation benötigt wird, kann daher erwartet werden. Vorangegangene Transplantationen haben gezeigt, dass die A. facialis in der Lage ist das Gesichtstransplantat sicher zu versorgen und daher auf schwierige und tiefe Inzisionen mit Einschluss der A. temporalis, deren Manipulation häufig zu Blutungskomplikationen führt, verzichtet werden kann [21]. Diese Meinung vertreten jedoch nicht alle Autoren [22]. Einige Arbeitsgruppen empfehlen bilaterale A. carotis externa Anastomosen, die jedoch gewisse Risiken bergen. Zum einen stellt die
Zunge auf Basis anatomischer Studien aufgrund fehlender Anastomosen zu Ästen der A. carotis interna ein Endorgan des Stromgebietes der A. carotis externa und ihrer miteinander kommunizierenden Äste dar [23]. Diese Versorgung wird jedoch durch Manipulation an der A. carotis externa einem Risiko ausgesetzt. Zum anderen kann eine Gefäßanomalie im Bereich der A. ophthalmica oder deren Thrombosierung und gleichzeitiger Verschluss der A. carotis externa durch Manipulation zu einem akuten okulären ischämischen Syndrom führen, welches letztlich zur Erblindung führt [24,25]. Falls das Transplantat parietale und/oder temporale Kopfhaut beinhaltet, kann eine ausreichende Durchblutung durch Erhalten der A. transversalis faciei, die aus der $A$. temporalis superficialis entspringt und zahlreiche Anastomosen mit der A. facialis unterhält, erreicht werden [15]. Somit ist auch hier keine Anastomose auf Höhe der A. carotis externa zwingend erforderlich.

Obwohl über spontanes Wiederkehren von Sensibilität nach Gesichtstransplantationen berichtet wurde, scheint es im Hinblick auf Langzeitresultate sinnvoll zu sein möglichst viele sensible Nervenverbindungen wiederherzustellen. Die Frage, ob eine Verlängerung der Operationszeit durch Koaptation sensibler Nerven gerechtfertigt ist, konnte bis dato jedoch noch nicht abschließend geklärt werden. Die am häufigsten koaptierten sensiblen Nerven sind $N$. infraorbitalis und N. buccalis $[11,21,26]$. N. supraorbitalis, supratrochlearis, alveolaris inferior und auricularis stellen weitere, mit relativ geringem Aufwand erreichbare, sensible Äste dar, deren Koaptation vom Chirurgen in Erwägung gezogen werden sollte [15].

2 Nerven im Gesichts- und Halsbereich sorgen für die motorische Innervation. Zum einen der N. hypoglossus, der die Muskulatur der Zunge versorgt, zum anderen der N. facialis, der für die Versorgung der mimischen Gesichtsmuskulatur zuständig ist. Er tritt an der Schädelbasis aus dem Foramen stylomastoideum aus und zieht in einem Bogen nach vorne, bevor er in die Parotis eintritt und sich in seine oberflächlichen und tiefen Äste aufspaltet. Am vorderen Rand der Parotis verlassen die einzelnen Fazialisäste, namentlich N. temporalis (frontalis), N. zygomaticus, N. buccalis, N. marginalis mandibularis und N. cervicalis die Parotis und ziehen in Richtung ihrer Innervationsgebiete. Zwar ist die Dissektion und Koaptation auf Stammlevel schneller durchzuführen, jedoch bringt diese Technik einen wichtigen Nachteil mit sich. Sowohl Hirnnerven als auch periphere Nerven führen Faszikel, die im Verlauf ihre topografische Lage innerhalb der Nervens ändern [27]. Es muss daher ein besonderes Augenmerk auf die intraneurale Topografie gelegt werden, um eine mög-

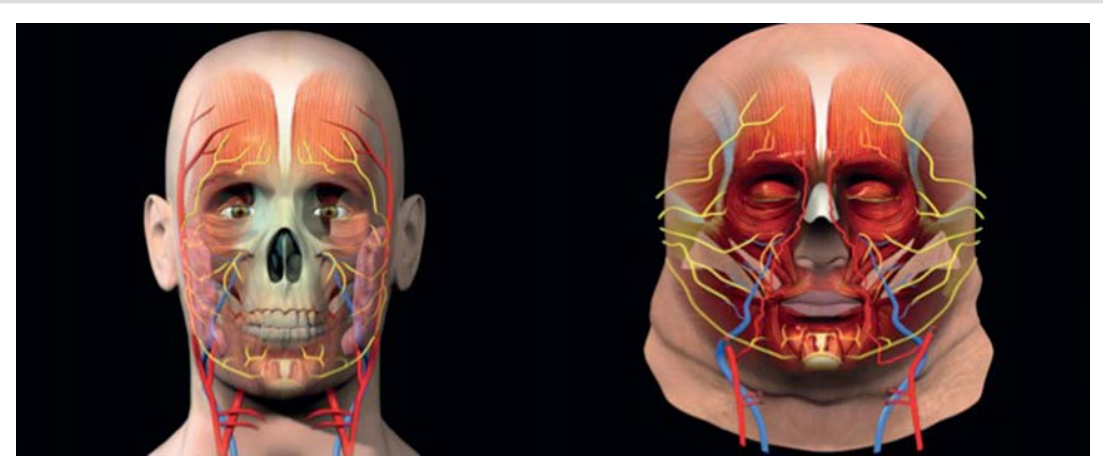

Abb. 3 links: reguläre Anatomie. Darstellung aller Strukturen, die zur Beschreibung der OP-Technik notwendig sind; rechts: Gesichtstransplantat mit allen Strukturen, deren Anastomosierung bzw. Koaptation für eine erfolgreiche Transplantation bei einem hypothetischen Patienten, mit Verlust aller vaskulären und nervalen Strukturen sowie Weichteile im Gesichtsbereich, notwendig sind. Publiziert in: Face transplantation. Pomahac et al. Curr Probl Surg. 2011 May; 48 (5): 293-357. doi: 10.1067/j.cpsurg.2011.01.003. PMID: 21463717

Sensorische Nerven (N. infraorbital, N. supraorbital, N. buccalis, N. mentalis)

Motorische Nerven (N. facials mit Ästen)

Arterien (A. carotis communis, interna und externa mit Ästen)

Venen ( $V$. jugularis intema und externa) 
lichst exakte Koaptation der einzelnen Faszikelgruppen zu gewährleisten und störende Synkinesien, die bei Reinnervation auftreten können, zu vermeiden. Auf dieser Grundlage lässt sich diskutieren, dass das proximale koaptieren von Nerven das Risiko von Synkinesien erhöht [28] und somit auch im Falle einer Gesichtstransplantation zu unvorhersehbarer Reinnervation und eingeschränkten funktionellen Ergebnissen führen kann. Daher sollte der Wiederanschluss möglichst distal der Parotis in Nähe zu den Zielmuskeln angestrebt werden. Hierzu ist auch die
Präparation der Fazialisäste des Empfängers zur sicheren Neurorrhaphie essentiell ( $\bullet$ Abb. 4).

\section{Immunsuppression und Abstoßung}

$\nabla$

Bis dato konnten die großen Fortschritte in der Immunsuppression bei Transplantation solider Organe noch nicht in den Bereich der VCA übertragen werden. Das mag daran liegen, dass
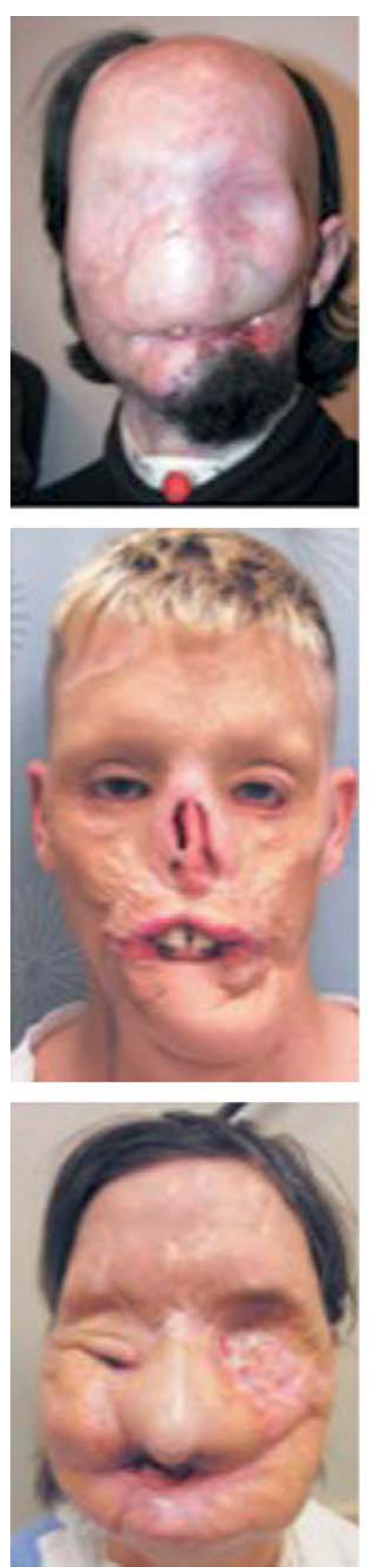
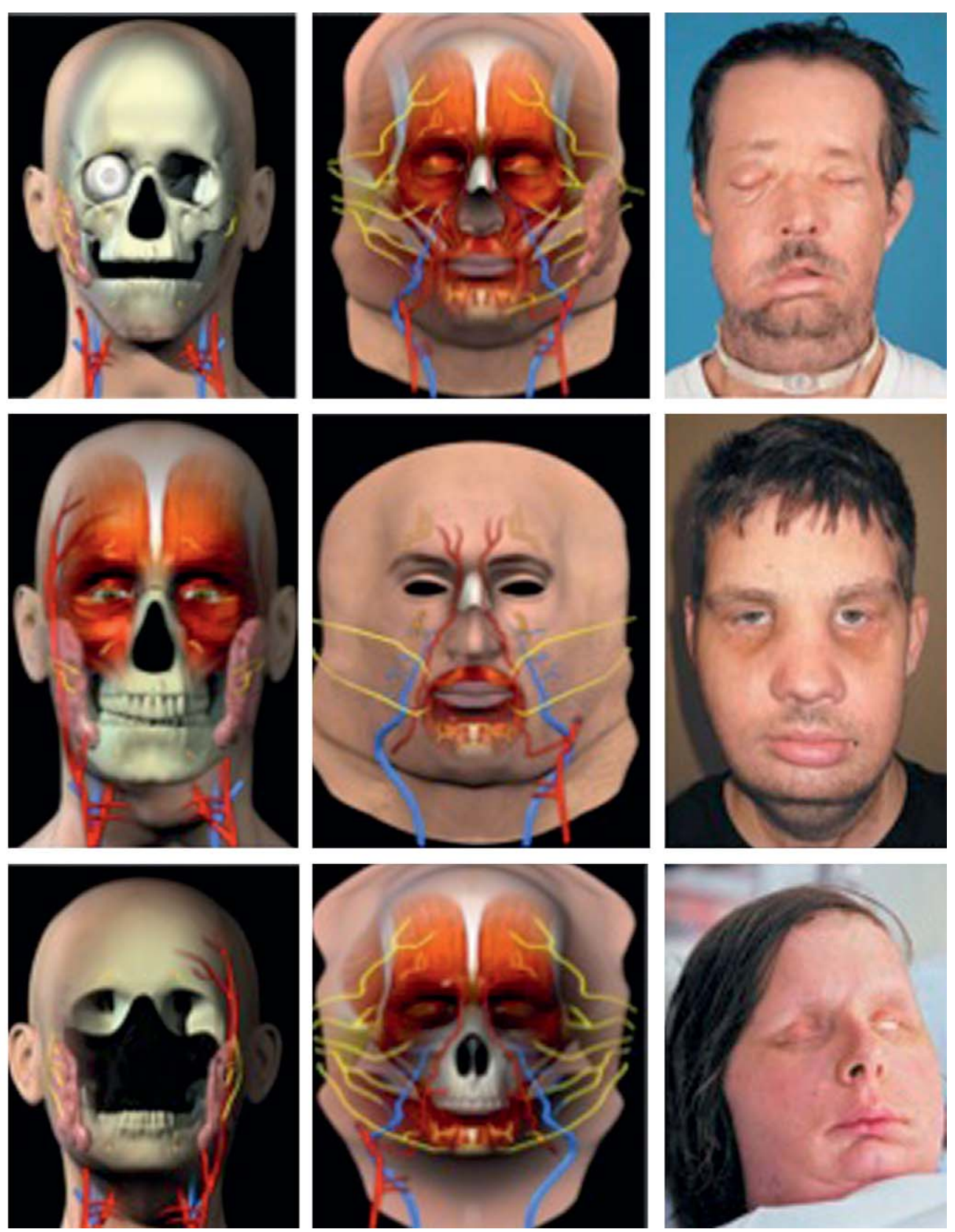

Sensorische Nerven (N. infraorbital, N. supraorbital, N. buccalis, N. mentalis)

Motorische Nerven (N. facials mit Ästen)

Arterien (A. carotis communis, interna und externa mit Ästen)

Venen (V. jugularis interna und externa)

Abb. 43 Patienten mit totaler Gesichtstransplantation: von links: präoperativ, Schema präoperative Empfängeranatomie, Schema der Transplantatanatomie (alle anastomosierten Gefäße und koaptierten Nerven dargestellt), Publiziert in: 3 patients with full facial transplantation. Pomahac et al., N Engl J Med. 2012 Feb 23; 366 (8): 715-22. doi: 10.1056/NEJMoa1111432. Epub 2011 Dec 28. PMID: 22204672 und Face transplantation. Pomahac et al. Curr Probl Surg. 2011 May; 48 (5): 293-357. doi: 10.1067/j.cpsurg.2011.01.003. PMID: 21463717. 
die Haut als das immunogenste Gewebe im menschlichen Körper angesehen wird. Jedoch befinden sich nun einige vielversprechende Immunsuppressiva wie Calcineurininhibitoren und antiproliferative Substanzen wie Mycophenolat Mofetil (MMF) nach erfolgreichen Versuchen im VCA Tiermodell [29] im klinischen Einsatz.

\section{Induktionstherapie \\ $\nabla$}

Der Großteil der Teams weltweit, die VCAs durchführen, verabreicht zur Induktion der immunsuppressiven Therapie Thymoglobulin in variierenden Dosen $(1,25-3,0 \mathrm{mg} / \mathrm{kgKg} /$ Tag für 3-10 Tage) [16,26,30-34]. Am Transplantationszentrum des Brigham and Women's Hospital wird perioperativ Thymoglobulin in einer Dosis von $1,5 \mathrm{mg} / \mathrm{kgKg} / \mathrm{Tag}$ für 4 Tage gegeben. Andere Zentren verabreichen perioperativ Anti-Interleukin-2 Rezeptor monoklonale Antikörper mit 1-2 Bolusinjektionen in den ersten Tagen bis Wochen postoperativ [35-38]. Interessanter Weise sind Tracheatransplantationen ohne Induktionstherapie mit Antikörpern ausgekommen, was möglicherweise an der fehlenden immunogenen Komponente Haut liegen mag [39].

\section{Erhaltungstherapie}

Die momentan gängigste Methode zur Erhaltung der Immunsuppression besteht aus einer Dreifachtherapie mit Tacrolimus, einer antiproliferativen Substanz und Corticosteroiden [40]. Am Brigham and Women's Hospital wird eine Kombination aus $1 \mathrm{~g}$ MMF präoperativ und anschließend $2 \mathrm{~g} /$ Tag plus Methylprednisolon $500 \mathrm{mg}$ i.v. perioperativ verabreicht. Das Methylprednisolon wird nachfolgend stetig reduziert und am 4 . postoperativen Tag gegen Prednison $40 \mathrm{mg}$ per os $2 \times$ täglich ausgetauscht. Ab Tag 5 beträgt die Dosis nur noch $20 \mathrm{mg}$ per os/Tag. Tacrolimus wird postoperativ mit dem Ziel eines stabilen Vollblutkonzentration von $10-15 \mathrm{ng} / \mathrm{ml}$ bis zum 3. Monat nach Transplantation verabreicht. Andere Arbeitsgruppen haben über vergleichbare Protokolle berichtet $[16,30-33,35,36,38]$. Im weiteren Verlauf wird die Gabe von Tacrolimus auf $6-8 \mathrm{ng} / \mathrm{ml}$ nach 6 Monaten und $4-6 \mathrm{ng} / \mathrm{ml}$ nach 12 Monaten reduziert. MMF wird nach Gabe der Initialdosis kontinuierlich zurückgefahren und Steroide konnten in allen gesichts- und handtransplantierten Patienten am Brigham and Women's Hospital zwischen Monat 1 und 12 nach Transplantation ausgeschlichen werden. Hierbei unterscheidet sich der Ansatz der steroidfreien Erhaltungstherapie von den Strategien anderer Zentren [30,33,35, 36, 41,42].

Einige Arbeitsgruppen haben postoperative hämatopoetische Stammzelltransplantationen mit anschließender serieller Photochemotherapie als Ergänzung zur Dreifachherapie durchgeführt $[16,43,44]$. Es konnte jedoch im Verlauf kein Chimärismus im peripheren Blut nachgewiesen werden [42]. Weitere alterna- tive Behandlungsansätze befassen sich mit Zelltherapie und Immunmodulation zur Toleranzinduktion und befinden sich momentan allesamt noch in experimentellen Stadien $[45,46]$.

\section{Abstoßung in VCA}

Nachdem zuvor über unterschiedliche Klassifikationssysteme publiziert worden war [47-50], trafen sich im Jahr 2007 Chirurgen, Pathologen und Grundlagenforscher zur „Ninth Banff Conference on Allograft Pathology“, um über die Notwendigkeit einer einheitlichen Skala zur Bewertung von Transplantatabstoßung in VCA zu beraten. Die Banff 2007 Klassifikation der akuten Abstoßung stellt das Ergebnis dieser Bemühungen dar und basiert auf der Lokalisation und Intensität des inflammatorischen Infiltrats [51]. Einige Zentren transplantieren zusätzlich zum eigentlichen Gesichtstransplantat einen freien fasziokutanen Radialislappen, genannt Sentinel Flap (SF) [20,34,52]. Dieser kann zur Überwachung von akuten Abstoßungsreaktionen und Abgrenzung zu anderen Ursachen erythematöser Effloreszenzen, wie bspw. Rosacea, nützlich sein [53]. Seine Relevanz gilt es in zukünftigen Studien zu eruieren. Episoden akuter Abstoßung treten in den ersten Monaten nach Gesichtstransplantation regelmäßig auf [16,26,32-36,38,39] und werden üblicherweise mit Steroid-Stosstherapie oder kurzzeitiger Dosissteigerung der Erhaltungstherapie behandelt [31-33]. Auch späte akute Abstossungen sind beobachtet worden. Die topische Applikation von Tacrolimus und Clobetasol hat bei der Behandlung von entzündlicher Dermatitis vielversprechende Resultate gezeigt [54] und ist aufgrund der zu erwartenden minimalen systemischen Toxizität von großem Interesse zur Behandlung akuter Abstoßung von VCAs. Chronische Abstossung wird generell durch Vaskulopathie mit myointimaler Proliferation, die mit Atrophie und Fibrose von Muskelgewebe, Haut und Hautanhangsgebilden einhergeht, definiert [55]. Bis dato sind keine verlässlichen Daten zu chronischer Abstoßung in VCA verfügbar und es ist ebenfalls keine Definition in die Banff Klassifikation aufgenommen worden. Die Abwesenheit chronischer Abstoßung mag auf die schnellere Diagnose und Behandlung akuter Abstoßungsepisoden zurückzuführen sein ( $\bullet$ Tab. 1).

\section{Diskussion \\ $\nabla$}

Die allgemeine Einstellung hinsichtlich der ethischen Vertretbarkeit hat sich mit der ersten erfolgreichen Gesichtstransplantation im Jahre 2005 innerhalb eines Jahrzehntes von Ablehnung der Prozedur hin zu einer generellen Akzeptanz weiterentwickelt [56]. Kürzlich wurde in den amerikanischen Medien in großem Stil darüber berichtet, dass Bürger in Zukunft bei der Entscheidung, ob sie Organe spenden möchten, Gesichts- und Handtransplantationen explizit ein- oder ausschließen sollen.

Tab. 1 Gradeinteilung akuter Abstoßung der Banff 2007 Klassifikation.

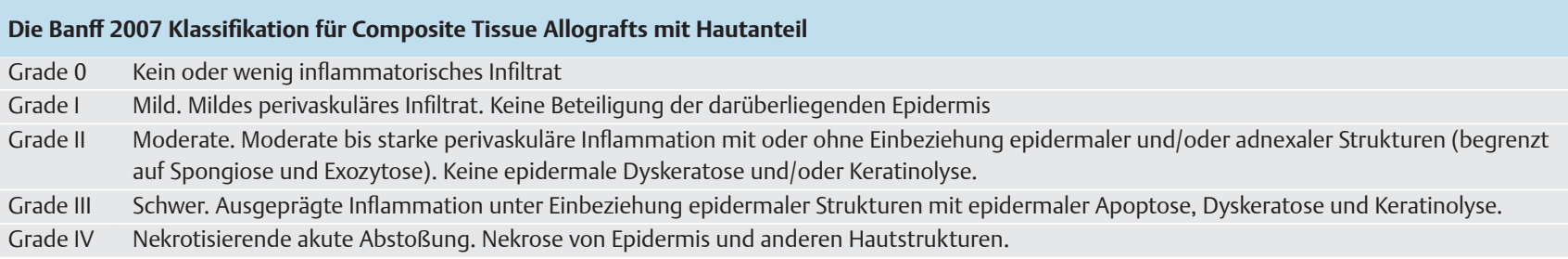


Somit werden Hand und Gesicht der gleiche Status wie soliden Organen zuerkannt und dabei auch den gleichen Regularien unterworfen. Dies kann in Zukunft die Familien von potentiellen Spendern bei der Entscheidung entlasten und konsekutiv zu einem Anstieg der Spenderzahlen führen.

Zum Großteil werden die Kosten für die Transplantationen aktuell über Forschungsetats (in den USA zum größten Teil von Geldern des Department of Defense) finanziert. Um auch in Zukunft die Ausgaben für Operationen, Nachsorge und immunsuppressive Medikation zu decken, sollte jedes Transplantationsteam ergebnisorientierte Studien publizieren. Positive Ergebnisse in Arbeiten zum funktionellen Outcome, moderaten Komplikationsraten, Patientenzufriedenheit und Kosteneffektivität im Vergleich zur konventionellen Rekonstruktion können einen wichtigen Schritt vom experimentellen Status von VCAs hin zur klinischen Etablierung bedeuten und somit auch die Krankenkassen zur Kostenübernahme bewegen.

Kontinuierliche Forschung in Richtung verbesserter Immunsuppression kann durch Verschiebung der Nutzen-Risiko-Grenze den Empfängerpool erweitern. Allerdings beschreibt die lebenslang notwendige Immunsuppression mit ihren potentiell letalen Nebenwirkungen nach wie vor den größten Gegenspieler der nicht lebensnotwendigen Gesichtstransplantation. Eine Weiterentwicklung der relativ unspezifischen Kriterien der Banff 2007 Klassifikation [13] und die Verbesserung von Monitormethoden, wie die Verwendung von SFs, kann zu einer effektiver angepassten Immunsuppression und Behandlung von Abstoßungsepisoden führen. Bis dato stehen keine ausreichenden Daten zur abschließenden Identifizierung der Mechanismen der chronischen Abstoßung in VCA zur Verfügung. Ihre Inzidenz scheint jedoch deutlich geringer als in der Transplantation solider Organe. Die Entwicklung nichtinvasiver Bildgebungstechniken wie die Ultraschallbiomikroskopie (UBM), die Intimaverdickungen sensitiver identifizieren kann, könnten zur frühzeitigen Diagnose und Behandlung chronischer Abstoßungsreaktionen beitragen [57].

Das Muskelgewebe des Transplantats limitiert die mögliche Ischämiezeit und somit den potentiellen Spenderpool. Die Minimierung des Ischämie-Reperfusionsschadens kann einerseits durch eine Verkürzung der tatsächlichen Ischämiezeit durch optimale Synchronisation der Operationsteams erreicht werden. Auf der anderen Seite steht die Verlängerung der möglichen Ischämiezeit, bspw. durch extrakorporale Perfusionsverfahren. Diese Methode findet in Bereich der Nierentransplantation bereits Anwendung, konnte jedoch für den Bereich der VCA noch nicht erfolgreich etabliert werden.

Nicht zuletzt gilt es die Operationstechniken weiter zu optimieren den und Zugang zu kompetenten Zentren zu verbessern.

\section{Schlussfolgerung}

$\nabla$

Die Gesichtstransplantation hat sich in kurzer Zeit zu einer klinisch relevanten Alternative zur herkömmlichen Rekonstruktion von ausgedehnten Gesichtsdefekten entwickelt. Mit der Publikation motivierender funktioneller und ästhetischer Ergebnisse hat sich auch die zunächst negative Einstellung hinsichtlich dieser nicht überlebensnotwendigen Transplantationen zum Positiven weiterentwickelt. Es bleiben jedoch weiterhin gravierende Risiken durch die notwendige lebenslange Immunsuppression bestehen. Intensive Forschung auf dem jungen Gebiet VCA kann zur weiteren Etablierung und Verbesserung des Nutzen-RisikoVerhältnisses dieser spannenden Verfahren beitragen.

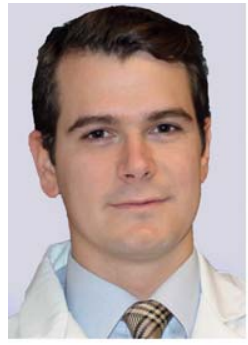

Maximilian Kückelhaus

Geboren 1985 in Wuppertal. 2006-2013

Studium der Humanmedizin an der

Universität Duisburg-Essen.

Promotionsarbeit zum Thema Wundheilungsförderung durch Applikation immunmodulierender Peptide. Assistenzarzt und wissenschaftlicher Mitarbeiter der Klinik für Plastische Chirurgie und Schwerbrandverletzte der BG Universitätsklinik Bergmannsheil Bochum (Direktor: Prof. Dr. M. Lehnhardt). Forschungsaufenthalte im R.E.W. Hancock Laboratory, Department of Microbiology and Immunology, University of British Columbia, Vancouver, Kanada und im Laboratory for Tissue Repair and Gene Therapy, Harvard Medical School, Boston, USA. Seit 2013 Postdoctoral Research Fellow am Center for Reconstructive and Restorative Surgery Research, Brigham and Women's Hospital, Harvard Medical School, Boston, USA.

Interessenkonflikt: Nein

\section{Literatur}

1 Brown JB, McDowell F. Massive Repairs of Burns with Thick Split-Skin Grafts: Emergency “Dressings” with Homografts. Ann Surg 1942; 115: 658-674

2 Brown JB, McDowell F. Epithelial Healing and the Transplantation of Skin. Ann Surg 1942; 115: 1166-1181

3 Tobin GR, Breidenbach WC $3^{\text {rd }}$, Ildstad ST et al. The history of human composite tissue allotransplantation. Transplant Proc 2009; 41: 466471

4 Harrison JH, Merrill JP, Murray JE. Renal homotransplantation in identical twins. Surg Forum 1956; 6: 432-436

5 Murray JE, Merrill JP, Dammin GJ et al. Study on transplantation immunity after total body irradiation: clinical and experimental investigation. Surgery 1960; 48: 272-284

6 Merrill JP, Murray JE, Takacs FJ et al. Successful transplantation of kidney from a human cadaver. JAMA 1963; 185: 347-353

7 Gilbert $R$. Transplant is successful with a cadaver forearm. Med Trib Med News 1964; 1964; 5: 20

8 Gilbert $R$. Hand transplanted from cadaver is reamputated. Med Trib Med News 1964; 1964; 5: 23

9 Dubernard JM, Owen E, Herzberg G et al. Human hand allograft: report on first 6 months. Lancet 1999; 353: 1315-1320

10 Dubernard JM, Owen E, Herzberg G et al. The first transplantation of a hand in humans. Early results. Chirurgie 1999; 124: 358-365 discussion 365-367

11 Devauchelle $B$, Badet $L$, Lengele $B$ et al. First human face allograft: early report. Lancet 2006; 368: 203-209

12 Murphy BD, Zuker RM, Borschel GH. Vascularized composite allotransplantation: An update on medical and surgical progress and remaining challenges. J Plast Reconstr Aesthet Surg 2013, doi:10.1016/j. bjps.2013.06.037

13 Sarhane KA, Tuffaha SH, Broyles JM et al. A Critical Analysis of Rejection in Vascularized Composite Allotransplantation: Clinical, Cellular and Molecular Aspects, Current Challenges, and Novel Concepts. Front Immunol 2013; 4: 406

14 Haxby JV, Hoffman EA, Gobbini MI. Human neural systems for face recognition and social communication. Biol Psychiatry 2002; 51: 59-67

15 Pomahac B, Pribaz J. Facial composite tissue allograft. J Craniofac Surg 2012; 23: 265-267

16 Dubernard JM, Lengele B, Morelon E et al. Outcomes 18 months after the first human partial face transplantation. N Engl J Med 2007; 357: 2451-2460

17 Groenewoud AF, Thorogood J. A preliminary report of the HTK randomized multicenter study comparing kidney graft preservation with HTK and EuroCollins solutions. HTK Study Group. Transpl Int 1992; 5 (Suppl 1): S429-S432 
18 Cavallari A, Cillo U, Nardo B et al. A multicenter pilot prospective study comparing Celsior and University of Wisconsin preserving solutions for use in liver transplantation. Liver Transpl 2003; 9: 814-821

19 Michel P, Vial R, Rodriguez C et al. A comparative study of the most widely used solutions for cardiac graft preservation during hypothermia. J Heart Lung Transplant 2002; 21: 1030-1039

20 Pomahac B, Pribaz JJ, Bueno EM et al. Novel surgical technique for full face transplantation. Plast Reconstr Surg 2012; 130: 549-555

21 Pomahac B, Lengele B, Ridgway EB et al. Vascular considerations in composite midfacial allotransplantation. Plast Reconstr Surg 2010; 125: 517-522

22 Meningaud JP, Benjoar MD, Hivelin $M$ et al. Procurement of total human face graft for allotransplantation: a preclinical study and the first clinical case. Plast Reconstr Surg 2010; 126: 1181-1190

23 Houseman ND, Taylor GI, Pan WR. The angiosomes of the head and neck: anatomic study and clinical applications. Plast Reconstr Surg 2000; 105: 2287-2313

24 Alizai AM, Trobe JD, Thompson BG et al. Ocular ischemic syndrome after occlusion of both external carotid arteries. J Neuroophthalmol 2005; 25: 268-272

25 Geibprasert S, Pongpech S, Armstrong D et al. Dangerous extracranialintracranial anastomoses and supply to the cranial nerves: vessels the neurointerventionalist needs to know. AJNR Am J Neuroradiol 2009; 30: $1459-1468$

26 Pomahac B, Pribaz J, Eriksson E et al. Restoration of facial form and function after severe disfigurement from burn injury by a composite facial allograft. Am J Transplant 2011; 11: 386-393

27 Jabaley ME, Wallace WH, Heckler FR. Internal topography of major nerves of the forearm and hand: a current view. J Hand Surg Am 1980; 5: 1-18

28 Piza-Katzer H, Balogh B, Muzika-Herczeg E et al. Secondary end-to-end repair of extensive facial nerve defects: surgical technique and postoperative functional results. Head Neck 2004; 26: 770-777

29 Ustuner ET, Zdichavsky M, Ren X et al. Long-term composite tissue allograft survival in a porcine model with cyclosporine/mycophenolate mofetil therapy. Transplantation 1998; 66: 1581-1587

30 Petruzzo P, Badet L, Gazarian A et al. Bilateral hand transplantation: six years after the first case. Am J Transplant 2006; 6: 1718-1724

31 Lantieri L, Meningaud JP, Grimbert P et al. Repair of the lower and middle parts of the face by composite tissue allotransplantation in a patient with massive plexiform neurofibroma: a 1-year follow-up study. Lancet 2008; 372: 639-645

32 Siemionow M, Papay F, Alam D et al. Near-total human face transplantation for a severely disfigured patient in the USA. Lancet 2009; 374: 203-209

33 Barret JP, Gavalda J, Bueno J et al. Full face transplant: the first case report. Ann Surg 2011; 254: 252-256

34 Pomahac B, Pribaz J, Eriksson E et al. Three patients with full facial transplantation. N Engl J Med 2012; 366: 715-722

35 Breidenbach WC, Gonzales NR, Kaufman CL et al. Outcomes of the first 2 American hand transplants at 8 and 6 years posttransplant. J Hand Surg Am 2008; 33: 1039-1047

36 Guo S, Han Y, Zhang X et al. Human facial allotransplantation: a 2-year follow-up study. Lancet 2008; 372: 631-638

$37 \mathrm{Hu} \mathrm{W,} \mathrm{Lu} \mathrm{J,} \mathrm{Zhang} L$ et al. A preliminary report of penile transplantation. Eur Urol 2006; 50: 851-853
38 Jones JW, Gruber SA, Barker JH et al. Successful hand transplantation. One-year follow-up. Louisville Hand Transplant Team. N Engl J Med 2000; 343: 468-473

39 Delaere $P$, Vranckx J, Verleden $G$ et al. Tracheal allotransplantation after withdrawal of immunosuppressive therapy. N Engl J Med 2010; 362: 138-145

40 Hautz T, Brandacher G, Zelger B et al. Immunologic aspects and rejection in solid organ versus reconstructive transplantation. Transplant Proc 2010; 42: 3347-3353

41 Lantieri L, Hivelin M, Audard V et al. Feasibility, reproducibility, risks and benefits of face transplantation: a prospective study of outcomes. Am J Transplant 2011; 11: 367-378

42 Petruzzo P, Testelin S, Kanitakis J et al. First human face transplantation: 5 years outcomes. Transplantation 2012; 93: 236-240

43 Andreu G, Leon A, Heshmati F et al. Extracorporeal photochemotherapy: evaluation of two techniques and use in connective tissue disorders. Transfus Sci 1994; 15: 443-454

44 Ciancio G, Miller J, Garcia-Morales RO et al. Six-year clinical effect of donor bone marrow infusions in renal transplant patients. Transplantation 2001; 71: 827-835

45 Gorantla VS, Brandacher G, Schneeberger $S$ et al. Favoring the riskbenefit balance for upper extremity transplantation - the Pittsburgh Protocol. Hand Clin 2011; 27: 511-520 ix-X

46 Gorantla VS, Schneeberger S, Brandacher G et al. T regulatory cells and transplantation tolerance. Transplant Rev (Orlando) 2010; 24: 147-159

47 Bejarano PA, Levi D, Nassiri $M$ et al. The Pathology of full-thickness cadaver skin transplant for large abdominal defects: a proposed grading system for skin allograft acute rejection. Am J Surg Pathol 2004; 28: $670-675$

48 Cendales LC, Kirk AD, Moresi JM et al. Composite tissue allotransplantation: classification of clinical acute skin rejection. Transplantation 2006; 81: 418-422

49 Kanitakis J, Petruzzo P, Jullien D et al. Pathological score for the evaluation of allograft rejection in human hand (composite tissue) allotransplantation. Eur J Dermatol 2005; 15: 235-238

50 Schneeberger S, Kreczy A, Brandacher G et al. Steroid- and ATG-resistant rejection after double forearm transplantation responds to Campath1H. Am J Transplant 2004; 4: 1372-1374

51 Cendales LC, Kanitakis J, Schneeberger S et al. The Banff 2007 working classification of skin-containing composite tissue allograft pathology. Am J Transplant 2008; 8: 1396-1400

52 Diaz-Siso JR, Bueno EM, Sisk GC et al. Vascularized composite tissue allotransplantation - state of the art. Clin Transplant 2013; 27: 330-337

53 Knoll BM, Hammond SP, Koo S et al. Infections following facial composite tissue allotransplantation - single center experience and review of the literature. Am J Transplant 2013; 13: 770-779

54 Sehgal VN, Srivastava G, Dogra S. Tacrolimus in dermatology-pharmacokinetics, mechanism of action, drug interactions, dosages, and side effects: part I. Skinmed 2008; 7: 27-30

55 Schneeberger S, Ninkovic M, Piza-Katzer $H$ et al. Status 5 years after bilateral hand transplantation. Am J Transplant 2006; 6: 834-841

56 Kiwanuka H, Bueno EM, Diaz-Siso JR et al. Evolution of ethical debate on face transplantation. Plast Reconstr Surg 2013; 132: 1558-1568

57 Kaufman CL, Ouseph R, Blair B et al. Graft vasculopathy in clinical hand transplantation. Am J Transplant 2012; 12: 1004-1016 